\title{
Ultrafast Propulsion of Water Nanodroplets on Patterned Graphene
}

\author{
Papadopoulou, Ermioni; Megaridis, Constantine M.; Walther, Jens H.; Koumoutsakos, Petros
}

Published in:

ACS Nano

Link to article, DOI:

10.1021/acsnano.9b00252

Publication date:

2019

Document Version

Peer reviewed version

Link back to DTU Orbit

Citation (APA):

Papadopoulou, E., Megaridis, C. M., Walther, J. H., \& Koumoutsakos, P. (2019). Ultrafast Propulsion of Water Nanodroplets on Patterned Graphene. ACS Nano, 13(5), 5465-5472. https://doi.org/10.1021/acsnano.9b00252

\section{General rights}

Copyright and moral rights for the publications made accessible in the public portal are retained by the authors and/or other copyright owners and it is a condition of accessing publications that users recognise and abide by the legal requirements associated with these rights.

- Users may download and print one copy of any publication from the public portal for the purpose of private study or research.

- You may not further distribute the material or use it for any profit-making activity or commercial gain

- You may freely distribute the URL identifying the publication in the public portal

If you believe that this document breaches copyright please contact us providing details, and we will remove access to the work immediately and investigate your claim. 


\title{
Ultrafast Propulsion of Water Nano-Droplets on Patterned Graphene
}

\author{
Ermioni Papadopoulou, ${ }^{\dagger}$ Constantine M. Megaridis, ${ }^{\ddagger}$ Jens H. Walther, ${ }^{\dagger}$, and
} Petros Koumoutsakos*,†

†Computational Science and Engineering Laboratory, ETH Zürich, Zürich, CH-8092, Switzerland

$\ddagger$ Department of Mechanical and Industrial Engineering, University of Illinois at Chicago, Chicago, Illinois 6060\%, USA

【Department of Mechanical Engineering, Technical University of Denmark, 2800 Kongens Lyngby, Denmark

E-mail: petros@ethz.ch

\begin{abstract}
The directed transport of liquids at the nanoscale is of great importance for nanotechnology applications ranging from water filtration to the cooling of electronics and precision medicine. Here we demonstrate such unidirectional, pumpless transport of water nanodroplets on graphene sheets patterned with hydrophilic/phobic areas inspired by natural systems. We find that spatially-varying patterning of the graphene surfaces can lead to water transport at ultrafast velocities, far exceeding macroscale estimates. We perform extensive molecular dynamics simulations to show that such high transport velocities $\left(\mathcal{O}\left(10^{2} \mathrm{~m} / \mathrm{s}\right)\right)$ are due to differences of the advancing and receding contact angles of the moving droplet. This contact angle hysteresis and the ensuing transport depend on the surface pattern and the droplet
\end{abstract}


size. We present a scaling law for the driving capillary and resisting friction forces on the water droplet, and use it to predict nano-droplet trajectories on a wedge-patterned graphene sheet. The present results demonstrate that graphene with spatially-variable wettability is a potent material for fast and precise transport of nano-droplets with significant potential for directed nanoscale liquid transport and precision drug delivery.

\section{Keywords}

nano-droplets, graphene, nanofluidics, wettability gradient, wettability patterning

The structural patterning of surfaces is broadly encountered in natural systems, implying possible functional advantages for the organisms and their interactions with their environment. Nano- and micro- structural and chemical surface patterns are encountered on insect bodies and plant leaves, and are frequently associated with the vital functions of organisms. $^{1,2}$ In turn, the interactions of natural nano-/micro-structures with liquids serve as inspiration for engineered surfaces meant to increase transport efficiency, liquid collection or condensation and heat transfer of micro- and nano-droplets. ${ }^{3-9}$ A prominent example is the water-collection system of the Namib desert beetle, exhibiting surfaces exploited for water collection and directional transport. ${ }^{10}$ Experimental studies inspired by these surfaces have successfully demonstrated the water harvesting mechanisms in the microscale regime. ${ }^{11,12}$ Another example involves the structure of the vein network of the banana tree leaves, that has inspired the construction of patterned hydrophilic surfaces for microscale systems of

higher heat transfer efficiency. ${ }^{13-15}$ The effect of wettability patterning on heat transfer ${ }^{16}$ was also investigated by studies that were focused on enhancing the cooling efficiency of microelectronic devices by guiding water on a hydrophilic wedge-shaped track surrounded by superhydrophobic terrains. ${ }^{17-19}$

The potential of wettability gradients for enhanced uni-directional liquid transport at the 
nano- and micro-scale has received significant attention for fast drug delivery, efficient heat transport ${ }^{20,21}$ and electricity generation; ${ }^{22}$ such gradients are considerably easier to manufacture than other structural patterns. ${ }^{8}$ The static contact angle exhibited by water droplets on graphene is one of the common fundamental configurations for comparing experimental and computational studies and validating MD potentials. ${ }^{23-27}$ In turn, MD simulations of water droplet transport on graphene surfaces or channels with a linear wettability gradient ,26,28,29 have shown that a droplet can maintain unidirectional as well as non-linear trajectories. ${ }^{28}$ Experimental studies have revealed the advantages of a wedge-shaped hydrophilic track for pumpless, rapid, uni-directional transport. ${ }^{30,31}$

Here, we examine graphene surfaces with patterned nano-scale wettability domains and show that such surfaces induce ultrafast unidirectional transport of water nano-droplets. We compute water droplet velocities of the order of $10^{2} \mathrm{~m} / \mathrm{s}$ based on experimentally-validated variations of surface energy corresponding to contact angles representing superhydrophobic $\left(\theta \geq 150^{\circ}\right)^{11,18}$ or superhydrophilic $\left(\theta \approx 0^{\circ}\right)^{14}$ surfaces. High transport velocities have been reported previously by few studies investigating propulsion due to chemical ${ }^{26-28}$ or large thermal gradients. ${ }^{32,33}$ Notably, the observed velocities are two orders of magnitude higher than the highest reported velocities of water in nanochannels and most studies featuring surface energy gradients. ${ }^{34-36}$ Our work is inspired by studies on water behaviour on microscale patterned surfaces ${ }^{17}$ that aimed to exploit surface patterning inspired by the Namib beetles and banana leaves.

At the nanoscale, we find that the effects of wettability differences are non-linearly accentuated. In addition, we find that there is a strong influence of the geometrical wettability patterning (shape and gradients) of the graphene surface on the nano-droplets transport. We evaluate the dependence of the induced transport velocities on the relative droplet size, and attribute the ultrafast movement of the water droplets to the respective contact angle differences across the patterned surface. Finally, we derive a scaling law for the normalized friction coefficient of water on a graphene surface of variable wettability. We use this law 
to construct an analytical model describing the driving forces of the droplet motion on a wedge-patterned substrate. The analytical model is shown to predict reliably the results of the computationally-intensive MD simulations. We expect that this law can be used for optimization of tapered-patterned surfaces in diverse applications, such as efficient drug delivery ${ }^{11}$ and cooling of microelectronics. ${ }^{18}$

\section{Results and Discussion}

We examine self-propulsion of nano-droplets on graphene surfaces patterned with the schemes shown in Fig. 1. We perform MD simulations of water droplets placed on double-layer graphene, patterned either by a banded gradient scheme (Fig. 1a), or a hydrophilic tapered track laid in a hydrophobic background (Fig. 1b). The pattern in Fig. 1a is defined by the band width and wettability (each band has uniform wettability), or by the initial width and the angle of the wedge track, $\phi$ (Fig. 1b). We vary the wettability of the inner zone of the wedge-shaped track, tuning it to represent a zone of hydrophilic, neutral or hyrophobic interaction with water. The patterns are inspired by biological systems for water collection ${ }^{17}$ and directed fluid transport. ${ }^{15}$ As discussed below, the MD simulations reveal that these patterns induce motion of the water droplets with velocities up to two orders of magnitude higher than those observed in other nanoscale systems. ${ }^{37,38}$ We quantify the droplet transport by the position and velocity of the droplet's center-of-mass (CoM), $\mathrm{P}_{\mathrm{CoM}}$ and $\mathrm{V}_{\mathrm{CoM}}$, and the difference $(\Delta \theta)$ between the contact angles of the advancing and receding interfaces (see following sections and the Supporting Information (SI)). 


\section{Water droplets on two wettability patterns: Uniform gradient $v s$. wedge track}

We compare the nano-droplet motion on surfaces with a uniform wettability gradient and a wedge-shaped track (Fig. 1a and b, respectively). We observe two distinct behaviors characterized by markedly different values of $\Delta \theta$, as shown in the starting and final side view of each droplet over the corresponding patterned sheet (Fig. 1c and d, respectively). Over the wedge-patterned surface, $\Delta \theta$ decreases steadily after the droplet settles on the surface, eventually falling below $5^{\circ}$ (Fig. 1f). In contrast, $\Delta \theta$ remains almost constant on the uniform gradient surface (Fig. 1f).

In the wedge-shape pattern, the droplet initially accelerates nearly at the same rate as on the gradient surface, but, soon, under the resistance of interfacial friction, it decelerates and, thus, its maximum attained velocity is below that on the uniform-gradient surface (Fig. 1e). In the following sections, we investigate the effects of the track's geometry on the droplet trajectory, with the aim to maximize the induced CoM droplet velocity.

We note that the initial droplet used for all simulations in this subsection is equilibrated on a "neutral" wettable surface with $\epsilon_{C O}=0.09369 \mathrm{kcal} / \mathrm{mol}$ and equilibrium contact angle $\theta=89^{\circ}$

Table 1: Values of the interaction strength between water and graphene atoms used in the different stripe zones of the wettability-gradient surface shown in Fig. 1a. The most hydrophobic (zone 1) and most hydrophilic (zone 7) values of this table are the ones used for the two regions in the wedge-shape patterned graphene surface in Fig. 1b

\begin{tabular}{l|ccccccc} 
Zone & 1 & 2 & 3 & 4 & 5 & 6 & 7 \\
\hline$\epsilon_{C O}[\mathrm{kcal} / \mathrm{mol}]$ & 0.05994 & 0.07494 & 0.08994 & 0.10494 & 0.11994 & 0.12994 & 0.13864 \\
Contact angle & $121^{\circ}$ & & & & & & $51^{\circ}$ \\
\hline
\end{tabular}



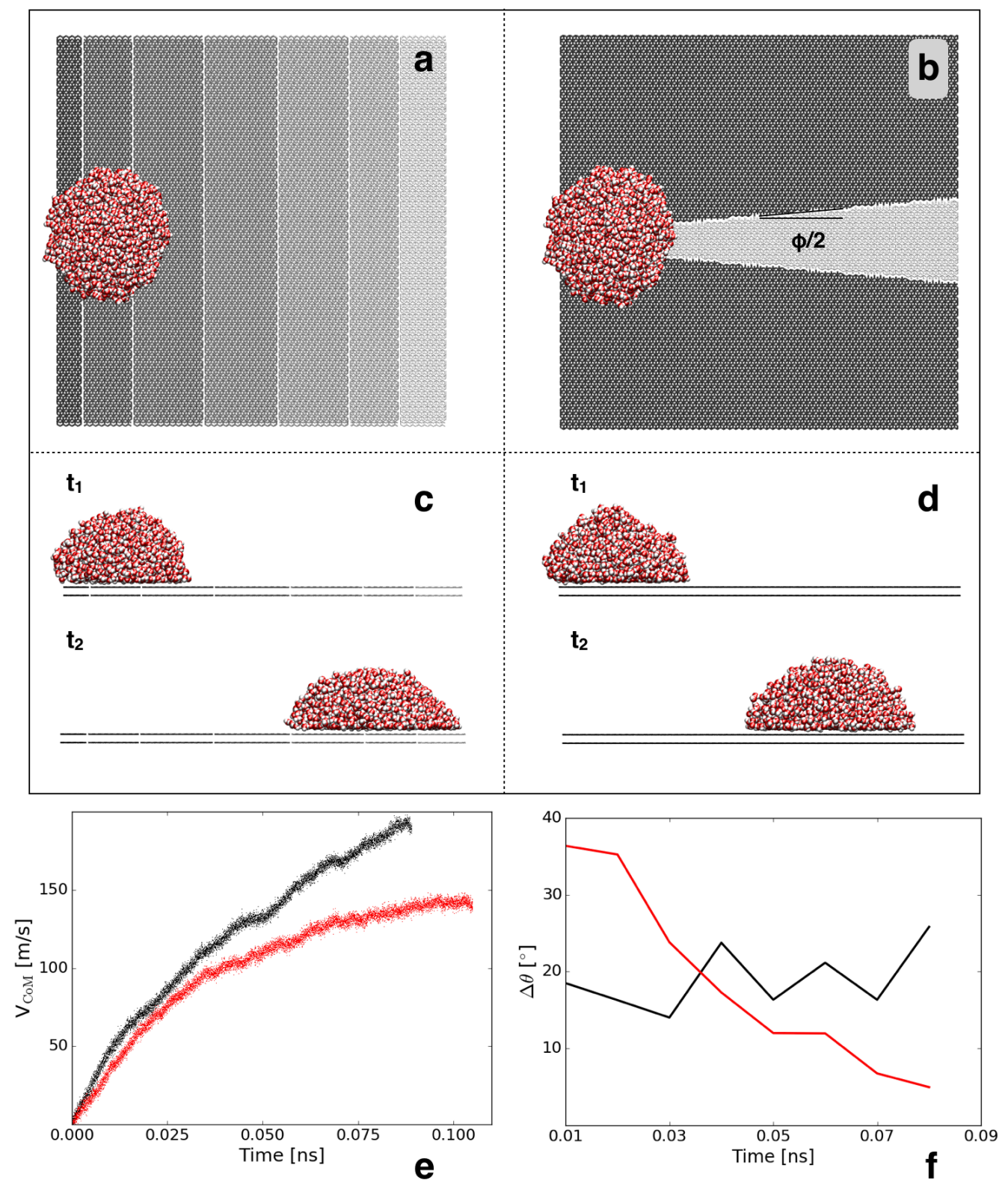

Figure 1: Subfigures (a-d): Geometry details of the compared wettability patterns: (a) Linear wettabilitygradient surface with values of the interaction strength between water and carbon, $\epsilon_{C O}$, varying for the zones from left to right as given in Table 1, (b) Wedge-shaped wettability patterned surface, which consists of two zones with $\epsilon_{C O}$ corresponding to the most hydrophobic (black colored zone) and most hydrophilic (silver colored zone) values in Table 1. (c) and (d) show side views of typical droplets at two time instances, $t_{1}$ and $t_{2}$ during their motion over the surfaces shown in (a) and (b), respectively. Subfigures (e-f): (e) Temporal comparison of the Center-of-Mass velocity $\left(\mathrm{V}_{\mathrm{CoM}}\right)$ of the droplets on a wedge track and a uniform gradient surface. (f) Difference of advancing and receding contact angles (contact angle hysteresis) during movement of water droplets placed on the gradient or the wedge-track surface. Black curves are for the gradient surface; Red curves are for the wedge-patterned surface. 


\section{Effect of structural variations of the graphene substrate}

Effect of wettability contrast of wedge-track. The wettability contrast of the wedgetrack patterned surface is critical to the droplet transport characteristics (Fig. 2a). For all cases in this section, the wedge (silver-colored in Fig. 1b) is hydrophilic, with a constant interaction strength $\epsilon_{C O}=0.13864[\mathrm{kcal} / \mathrm{mol}]$ that corresponds to an equilibrium contact angle $\theta=51^{\circ}$. The interaction strength $\epsilon_{C O}$ of the outer, less-wettable zone (black-colored in Fig. 1b) is varied, as shown in Table 2. The original wedge pattern, discussed in the previous section, corresponds to case 2 in Table 2. We observe that a terminal CoM droplet velocity, $\mathrm{V}_{\mathrm{CoM} \text { max }}$, is reached only for the more hydrophilic cases 03 and 04 , while the velocity in case 05 peaks early and declines steadily thereafter. As expected, $\mathrm{V}_{\mathrm{CoM} \text {,max }}$ declines with decreasing wettability contrast (lower $\theta_{\text {out }}$ or higher $\epsilon_{C O}$ ) (see Fig. 2a).

Table 2: Values of the interaction strength $\epsilon_{C O}$ between water molecules and graphene atoms in the outer zone of the wedge-shaped pattern, denoting the different wedge cases in Fig. 2. These values designate $\theta_{\text {out }}$ for the five cases listed. The value of $\epsilon_{C O}$ for the inner (more wettable) zone in all cases remains constant $\epsilon_{C O}=0.13864 \mathrm{kcal} / \mathrm{mol}$ and corresponds to $\theta=51^{\circ}$.

\begin{tabular}{l|ccccc} 
Wedge Case & 1 & 2 & 3 & 4 & 5 \\
\hline Outer zone $\epsilon_{C O}[\mathrm{kcal} / \mathrm{mol}]$ & 0.03994 & 0.05994 & 0.07994 & 0.09994 & 0.11994 \\
Water contact angle $\theta_{\text {out }}\left[{ }^{\circ}\right]$ & 147 & 121 & 107 & 88 & 68 \\
\hline
\end{tabular}

Effect of wedge-track angle. The geometry of the wedge track, and more specifically the wedge angle, $\phi$, greatly affects the early stages of the motion of the water droplet (Fig. 2b). We consider three wedge tracks with angles: $\phi=5^{\circ}, 10^{\circ}$ and $25^{\circ}$. We observe that the droplet CoM velocity peaks at a terminal value, whereas for the two higher values of $\phi$ it starts to decline in a shorter time, due to extensive friction in the larger hydrophilic zone. The acceleration of the droplet rises with increasing $\phi$, as expected. ${ }^{17}$ We emphasize that for both $\phi=10^{\circ}$ and $25^{\circ}$ wedge tracks, the initial acceleration is higher than the constant-gradient pattern, although the sustained influence of the wettability gradient eventually causes the droplet on the gradient surface to move faster. Note, again, that maximum droplet speeds exceed $150 \mathrm{~m} / \mathrm{s}$. We note that in previous experimental work with mm-scale 
tracks and droplets, the track angle $\phi$ has been limited to values well below $10^{\circ}$ due to the rapid widening of the track at high values of $\phi .^{17,30}$
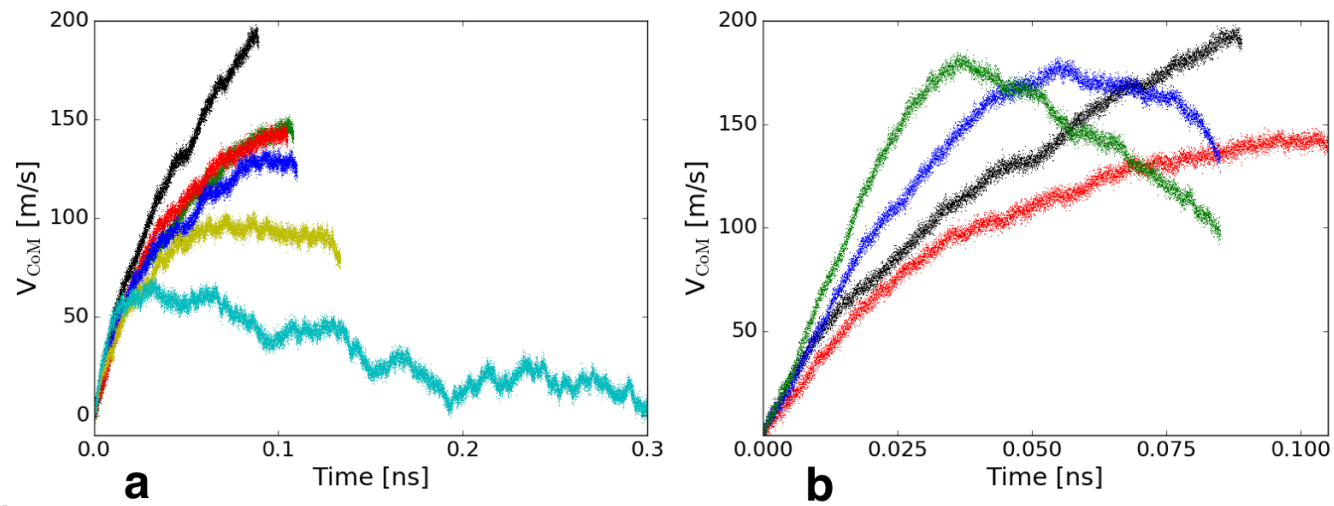

Figure 2: a) Center-of-Mass (CoM) velocity $\mathrm{V}_{\mathrm{CoM}}$ vs. time for water droplets moving on graphene surfaces with a straight wettability gradient pattern and wedge-shape patterns with the wettabilities of the outer zone listed in Table 2. The black curve is for the uniform-gradient surface; The colored curves refer to different wedge-shaped patterned surfaces, corresponding to the cases listed in Table 2: Green curves refer to case 1 $\left(\theta_{\text {out }}=147^{\circ}\right)$; Red to case $2\left(\theta_{\text {out }}=121^{\circ}\right)$; Blue to case $3\left(\theta_{\text {out }}=107^{\circ}\right)$; Yellow to case $4\left(\theta_{\text {out }}=88^{\circ}\right)$; Cyan to case $5\left(\theta_{\text {out }}=68^{\circ}\right)$. (b) Center-of-Mass $(\mathrm{CoM})$ velocity $\mathrm{V}_{\mathrm{CoM}}$ vs. time for the motion of water droplets on graphene surfaces with a straight gradient wettability pattern and a wedge-shape track with increasing wedge angles $\phi$. The black curve refers to the uniform-gradient surface; Colored curves refer to wedge-shaped patterned surfaces, with different wedge angles $\phi$ : Red for $\phi=5^{\circ}$; Blue for $\phi=10^{\circ}$; Green for $\phi=25^{\circ}$. The graphs extend up to the point when each droplet reached the end of the substrate. See more in Supporting Information (SI), Section 3.1.

\section{Effect of the droplet shape and size}

Effect of initial droplet shape. The structural characteristics of the water droplet at the instance it is brought in contact with the patterned surface have an effect on its subsequent motion (see SI Section 3.2.1 for more details). The initial behaviour of a spherical water droplet, that is first equilibrated in vacuum, has been found to depend on the pattern of the surface it is placed over (wedge-track or gradient). The water droplet structure is affected as it is lowered (by applying a constant velocity on water molecules) onto the patterned surface. The initially-spherical droplet approaching the wedge-patterned surface gets readily attached to the surface, on to the hydrophilic zone at the start of the track. Even though the terminal velocity of the droplet is lower in the case of the wedge pattern, the droplet reaches the end of the wedge-patterned substrate sooner than for the straight-patterned gradient 
surface (Fig. 3). We remark that the wedge track sets the fluid to a more vigorous motion and imparts initially higher acceleration than that observed on the gradient wettability surface. However, for the latter, the sustained influence of the wettability-gradient-induced force eventually raises the fluid velocity to levels beyond those attained on the wedge-shaped track.
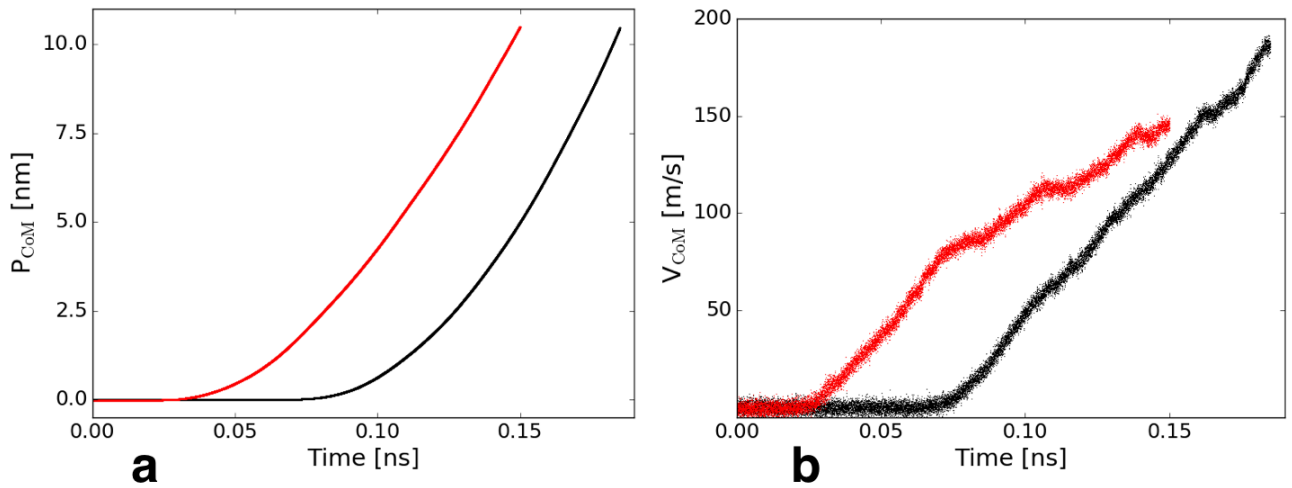

Figure 3: (a) Center-of-Mass $(\mathrm{CoM})$ position $\mathrm{P}_{\mathrm{CoM}}$, and (b) respective velocity $\mathrm{V}_{\mathrm{CoM}}$ vs. time for water droplets moving on graphene surfaces with a wettability gradient (Black curves) and the wedge-shaped track 02 in Table 2 (Red curves), using a spherical droplet equilibrated in vacuum. The graphs extend up to the point when each droplet reached the end of the substrate.

Droplet size effects on wedge-track transport. We equilibrate three droplets of different sizes on a neutrally-wettable surface (Table 3). The geometric parameters of the wedge-patterned surfaces, i.e. wedge angle, $\phi$, and initial width of the hydrophilic zone, are scaled according to the droplet size, so that the problem remains similar for every case (Table 3). The transport characteristics of the droplets along the track are only affected by the size of the droplet (see SI Section 3.2.2 for more details). For each case, the velocity initially rises under the influence of the Laplace pressure that forces the fluid onto the wettable track to minimize its energy. ${ }^{17}$ As the liquid moves to the wider portions of the track, the propelling force (due to the Laplace pressure) weakens, while the friction resistance rises. This competition results in the velocity peaking, and then declining, as the friction force keeps rising further (due to the fluid-solid contact area rising towards the wide end of the track). The maximum terminal velocity and how it varies with the droplet size is shown 
in Fig. 4. This trend is expected, as larger droplets have increased inertia as well as a larger contact area (i.e. higher frictional resistance) with the underlying wettable track.

Table 3: Values of the mean equilibration radius $R_{\text {equil }}$ of the contact interface of water and carbon, number of water molecules, equilibration contact angle $\theta_{\text {equil }}$, and corresponding initial width and angle of the wedge on the patterned surface. $\theta_{\text {equil }}$ here corresponds to the measured contact angle of the droplet when equilibrated prior to its release on the patterned graphene surface (see Methods section for more details).

\begin{tabular}{l|ccc} 
Case & 1 & 2 & 3 \\
\hline$\#$ of $\mathrm{H}_{2} \mathrm{O}$ molecules & 1474 & 3759 & 53331 \\
$R_{\text {equil }}[\AA]$ & 57 & 79 & 180 \\
$\theta_{\text {equil }}\left[^{\circ}\right]$ & 89 & 96 & 94 \\
Track starting width $[\AA]$ & 6.3 & 8.7 & 20.0 \\
Wedge angle $\phi\left[^{\circ}\right]$ & 1.6 & 2.2 & 5.0
\end{tabular}

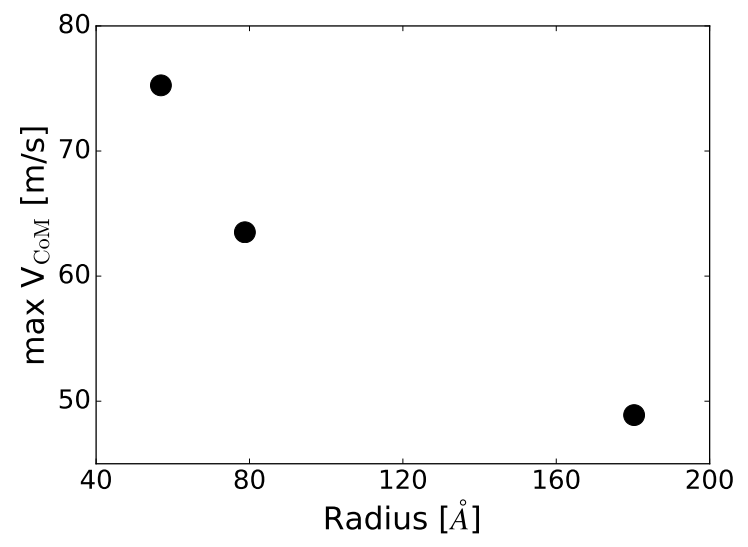

Figure 4: Maximum terminal velocity of a nano-droplet on a wedge-track versus droplet size.

\section{Interfacial friction coefficient}

We have performed a systematic study to extract the area-normalized friction coefficient of water droplets ${ }^{39}$ moving on surfaces of different wettabilities. This quantity is used in the following section to determine the friction force applied at the interface between the droplet and the patterned substrate. For each surface with different wettability, we apply a constant force on all water molecules and let the droplet move until the interfacial friction 
force between water and substrate equals the driving force (i.e. the droplet attains a steady velocity). We then correlate the steady (terminal) velocity of the droplet, $U$, and the friction force, $F_{f r}$, which is the pair force between water and the surface, and equals the applied force at equilibrium. Plotting the friction force versus the steady terminal velocity for different values of the applied driving force (Fig. 5a), we extract the friction coefficient for that surface via a linear fit of the data. The friction coefficient is defined as $\mu=\frac{F_{f r}}{U A}$, where $A$ is the wetted area of the substrate. Finally, collecting the results from the cases with different wettabilities, we deduce a quadratic relation between the friction coefficient and the surface wettability, as seen in Fig. 5b, which can provide estimates for the friction coefficient for all wettability values over the tested range.
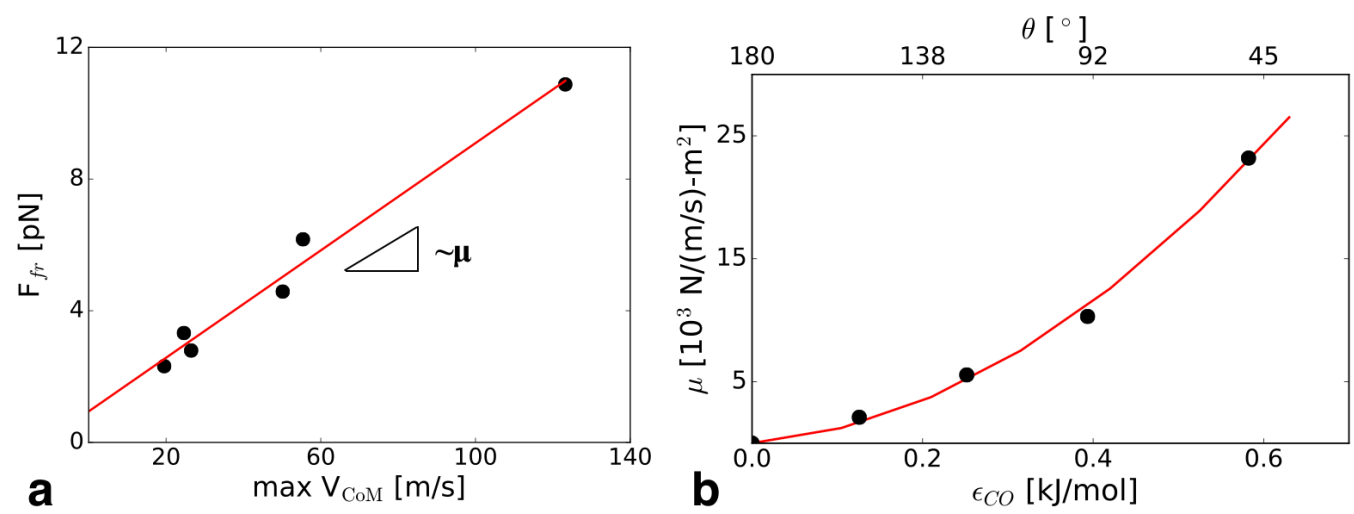

Figure 5: (a) Friction force $F_{f r}$ versus terminal velocity for surface wettability corresponding to $\epsilon_{C O}=$ $0.5994 \mathrm{kcal} / \mathrm{mol}$. The extracted friction coefficient $\mu$ is analogous to the slope of the line fitting the force data. For this case, we find $\mu=5554 \mathrm{~N} /(\mathrm{m} / \mathrm{s})-\mathrm{m}^{2}$. (b) Friction coefficient $\mu$ versus surface wettability, as defined by the interaction strength $\epsilon_{C O}$. A quadratic fit is found to describe the relation of $\mu$ and $\epsilon_{C O}$ : $\mu=a \epsilon_{C O}^{2}+b \epsilon_{C O}+c$, with $a=57696, b=5726$ and $c=0$. The top x-axis denotes the respective contact angle $\theta$ values corresponding to the ticks of the wettability $\epsilon_{C O}$ axis.

\section{Analysis of droplet transport on wedge-shape patterned surfaces}

We now establish a simple, analytical model for the forces acting on the water droplet when it is moving on a wedge-patterned surface. We establish the driving force induced by the wettability contrast between the two zones of the wedge track, $\mathbf{F}_{\mathbf{d r}}$, and the friction force between the droplet and the underlying surface, $\mathbf{F}_{\mathrm{fr}}$, which hinders the droplet's motion 
(Fig. 6a). The propelling force $\mathbf{F}_{\text {fr }}$ is linearly dependent on the wettability jump $S$ along the separation line of the two zones, $S=\frac{\mathrm{d} \cos (\theta)}{\mathrm{d} \chi}$, where $\theta$ is the contact angle of each wettable region and $\chi$ defines the direction perpendicular to the separating line between the two zones. Thus, in the direction of motion, $x: F_{d r, x}=\gamma A S \sin \phi / 2$, where $\gamma$ is the water surface tension, ${ }^{40}$ and $A$ the total contact area of the droplet with the substrate that contributes to the motion actuation. The wettability of the outer hydrophobic and inner hydrophilic zones is characterized by the contact angles $\theta_{A}$ and $\theta_{B}$, respectively. The friction force at the direction of the droplet's motion, is calculated as the sum of the friction forces on the two distinct zones of wettability: each force is calculated as the product of the friction coefficient of each wettability zone ( $\mu_{A}$ and $\mu_{B}$ for the hydrophobic and hydrophilic zone, respectively), as described in the previous section, with the droplet-wetted area $A$ of each zone, and the terminal velocity of the droplet, $U ; F_{f r}=\mu A U$. Further details about the analytical force evaluation can be found in SI Section 4.

The input to the analytical model is the initial position and velocity of the droplet from the MD simulation results. The output of the model defines the instant acceleration of the droplet and, in turn, the instantaneous velocity and position of the droplet on the track. We compare the evolution of the velocity of the droplet along its trajectory, as calculated from the analytical model, against the velocity evolution derived from the MD simulations. We find good agreement between the simulation and the model for the three droplet sizes in Table 3 (see Fig. 6b and SI Fig. S7 for all three sizes). We note that the current model matches the simulation results for a section of the overall trajectory, as our analysis does not account for end effects (i.e. the force induced by the wettability jump at the start of the wedge track or for the decelerating force when approaching the right periodic end of the substrate). Our analytical estimates indicate that the observed fast pumpless transport of water droplets at the nanoscale can be observed in experimental setups involving mm-scale droplets (see SI, Section 4). 


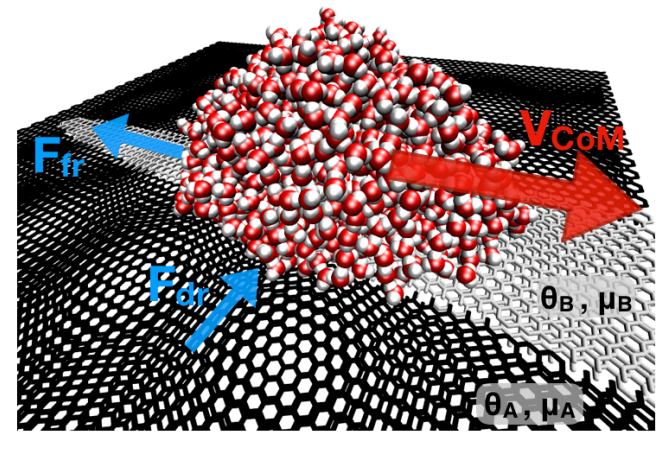

a

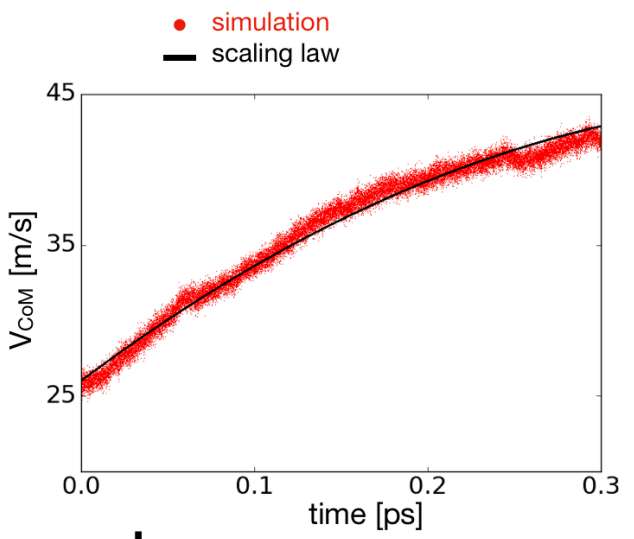

b

Figure 6: (a) Water-droplet transport over a graphene substrate in the direction of the velocity vector $V_{\mathrm{CoM}}$, under the influence of the capillary driving force, $\mathbf{F}_{\mathbf{d r}}$, and the resisting friction force, $\mathbf{F}_{\mathbf{f r}}$. The contact angles designating the wettability of the outer or inner zone of the wedge track are denoted as $\theta_{A}$ and $\theta_{B}$, respectively, whereas the respective friction coefficients are $\mu_{A}$ and $\mu_{B}$. (b) Comparison of the droplet velocity evolution from MD simulations (Red) and from the analytical model presented in this section (Black) for a droplet with $R_{\text {equil }}=180 \AA$ (case 3 in Table 3 ).

\section{Conclusions}

We present Molecular Dynamics simulations of ultra fast $\left(\sim \mathrm{O}\left(10^{2} \mathrm{~m} / \mathrm{s}\right)\right)$ transport of water nano-droplets on bio-inspired wettability patterns laid on graphene surfaces. We show that the motion of water droplets on gradient band-wettability patterns can be rationalized by the time evolution of the difference of advancing and receding contact angles. The water droplet transport on the wedge tracks is uni-directional with a terminal speed of transport that can be enhanced by changing the geometry of the track. We demonstrate the advantage of the wedge-track pattern over a straight gradient wettability pattern with respect to the directionality of the droplet motion. We identify the driving mechanisms for these phenomena and formulate an analytical model that evaluates the instantaneous acceleration of the droplet and could facilitate the design of such patterned surfaces. The results of this study can further assist ongoing research on spontaneous actuation of water transport due to interfacial phenomena, crucial for high-efficiency drug-delivery and ultrafast heat dissipation for nano- and micro-scale systems. Given the plethora of the available nanoscale fabrication 
methods to generate such wettability gradients, the present study supports the feasibility of moving minute amounts of fluids with very high velocities that are not attainable with macroscopic devices.

\section{Methods}

We perform MD simulations of water droplets placed on double-layer graphene, patterned either by a banded gradient scheme (Fig. 1a), or a hydrophilic pattered track laid in a hydrophobic background (Fig. 1b). The pattern in Fig. 1a is defined by the band width and wettability (each band has uniform wettability), or by the initial width and the angle of the wedge track, $\phi$ (Fig. 1b). We vary the wettability of the outer zone of the wedge-shaped track, tuning it to represent a zone of neutral or hyrophobic interaction with water. The inner zone of the wedge track remains hydrophilic at all simulations. For all simulation cases, the water droplet has been equilibrated on a graphene surface with a neutral wettability. We tune the wettability of the graphene surface by modifying the value of the Lennard-Jones interaction strength $\left(\epsilon_{C O}\right)$ between carbon and oxygen atoms. ${ }^{23,25}$ The baseline simulation setup uses a droplet of 1474 water molecules and is equilibrated on a "neutrally wettable" surface with interaction strength $\epsilon_{C O}=0.09369 \mathrm{kcal} / \mathrm{mol}$, corresponding to an equilibrium contact angle of $89^{\circ} .41$

All MD simulations are performed with the LAMMPS ${ }^{42}$ package. A timestep of 1 fs and periodic boundary conditions are applied to all simulations. The interactions of the water molecules are modeled with the TIP4P-Ew water model ${ }^{43}$ with molecular topology constraints applied with the SHAKE algorithm. ${ }^{44}$ For short-range interactions, we use a cutoff of $1.0 \mathrm{~nm}$ for the pair $\mathrm{C}^{-} \mathrm{O}^{45}$ and a cutoff of $0.9 \mathrm{~nm}$ for the pair O-O. ${ }^{46}$ From a sensitivity analysis for the settings of short-range interactions on our results, we find that the end-value of the CoM velocity of the droplet varies approximately $4-5 \%$ for a $10 \%$ variation of the cutoff distance (see SI, Section 1.3). For the computation of long-range electrostatic 
interactions, we set the cutoff distance at $0.85 \mathrm{~nm}^{43}$ and we use a particle-particle particlemesh (P3M) algorithm ${ }^{47}$ with a root-mean-squared error in the force calculation of $10^{-5}$.

The graphene atoms remain fixed for the majority of simulations, in order to eliminate further noise in the results due to phonon vibrations. ${ }^{48,49}$ We tested how the phonon excitation of the top layer of graphene would affect the results; we found that the end value of the CoM velocity of a water droplet on the graphene with the band gradient varies by $8 \%$, but only by $1.5 \%$ with the wedge pattern. We attribute this discrepancy to the spatial constraints the wedge pattern imposes on the droplet, enhancing its uni-directional translational motion (see SI, Section 1.5). We tune the wettability of the graphene surface by modifying the value of the Lennard-Jones interaction strength $\left(\epsilon_{C O}\right)$ between carbon and oxygen atoms. ${ }^{23,25}$ Visualizations were performed with the Visual Molecular Dynamics (VMD) package. ${ }^{50}$ Post-processing of the results has been partially performed using the MDAnalysis package. ${ }^{51}$

We build the various-sized graphene sheets with the WCCNT package, developed within the CSELab. ${ }^{52}$ The package works within the VMD software interface ${ }^{50}$ as a TCL plugin, and it integrates different routines to generate and analyze LAMMPS-compatible files of graphene sheets (GRSs) and carbon nanotubes (CNTs) with water. Preparation of the systems, including defining the various wettability zones on the graphene sheets, was also performed with TCL-scripting within the VMD package. The water droplets preparation is performed with the following procedure: For each case of droplet size, a water box is deposited on a graphene surface with the same wettability as the inner zone of the wedge track and is left to equilibrate to a droplet in an isothermal-isochoric (NVT) ensemble at $298 \mathrm{~K}$ for $2 \mathrm{~ns}$. The equilibration is over when the measured contact angle of the droplet does not change further. ${ }^{41}$

The majority of MD simulations are conducted with equilibrated water droplets, placed on patterned GRSs and left to move under the influence of interfacial forces (as described in SI, Section 4). For all simulations, the water molecules are kept at $298 \mathrm{~K}$, with the 
Nosé- Hoover thermostat, ${ }^{53}$ adjusting only the thermal component of temperature, which was found to be equivalent to employing an NVE ensemble ${ }^{54,55}$ (see SI, Section 1.4). The simulations performed in the Results section are summarized in SI, Section 2. For the friction coefficient study we apply a constant force on all oxygen atoms and let the droplet move until the interfacial friction force between water and substrate equals the driving force. We then measure the steady terminal velocity of the droplet, $U$, and the friction force, $F_{f r}$. This is the pair force between water and the surface and equals the applied force at equilibrium. We extract the friction coefficient by performing a set of 6 simulations, varying the applied force on the water molecules. This is repeated for 4 different values of surface wettability (varying the interaction strength $\epsilon_{C O}$ ), in order to construct the collective graph in Fig. 5b. This required $24 \mathrm{MD}$ simulations of 5 ns.

\section{Author Information}

\section{Corresponding author}

Petros Koumoutsakos, email: petros@ethz.ch

\section{Author contributions}

E.P., J.H.W., C.M.M. and P.K. designed the MD studies. E.P. performed the MD simulations and the analysis of the MD results. C.M.M. and P.K. coordinated the project and participated in the analysis of the results. J.H.W. participated in the analysis of the results. All authors contributed to writing and editing of the manuscript.

\section{Associated Content}

The authors declare no competing financial interest.

\section{Supporting Information}

This material is available free of charge via the Internet at http://pubs.acs.org. 
Droplet equilibration; change of surface wettability; effect of cutoff distance of short-range interactions; effect of thermostatting; effect of phonon excitation of the top graphene layer; simulation campaign; complementary graphs for the effects of structural variations of the graphene substrate on the water droplet transport; relation of difference of hysteresis and maximum achieved velocity of the droplet; complementary graphs for the effects of the shape and size of the droplet on its transport; analytical evaluation of forces.

\section{Acknowledgement}

This work is supported by the European Research Council Advanced Investigator Award (Grant No. 341117). We acknowledge the Swiss National Supercomputing Centre (CSCS) for computing time through project $\mathrm{CH} 7$.

\section{References}

1. Bourely, F. Hidden Beauty: Microworlds Revealed; Harry N. Abrams Inc.: New York, NY, 2002.

2. Ball, P. Patterns in Nature: Why the Natural World Looks the Way it Does; The University of Chicago Press: Chicago, IL, 2018.

3. Pulizzi, F.; Sun, W. Treating Water with Nano. Nat. Nanotechnol. 2018, 13, 633.

4. Nachtigall, W.; Blüchel, K. Das Grosse Buch der Bionik; Deutsche Verlags-Anstalt DVA: Stuttgart München, 2000.

5. Darhuber, A. A.; Troian, S. M. Principles of Microfluidic Actuation by Modulation of Surface Stresses. Annu. Rev. Fluid Mech. 2005, 37, 425-455.

6. Koch, K.; Barthlott, W. Superhydrophobic and Superhydrophilic Plant Surfaces: an Inspiration for Biomimetic Materials. Philos. T. R. Soc. A 2009, 367, 1487-1509.

7. Bhushan, B.; Jung, Y. C. Natural and Biomimetic Artificial Surfaces for Superhydrophobicity, SelfCleaning, Low Adhesion, and Drag Reduction. Prog. Mater. Sci. 2011, 56, 1-108. 
8. Xia, D.; Johnson, L. M.; López, G. P. Anisotropic Wetting Surfaces with One-Dimesional and Directional Structures: Fabrication Approaches, Wetting Properties and Potential Applications. Adv. Mater. 2012, 24, 1287-1302.

9. Song, Y.; Liu, Y.; Jiang, H.; Li, S.; Kaya, C.; Stegmaier, T.; Han, Z.; Ren, L. Bioinspired Fabrication of One Dimensional Graphene Fiber with Collection of Droplets Application. Sci. Rep. 2017, 7, 12056.

10. Parker, A. R.; Lawrence, C. R. Water Capture by a Desert Beetle. Nature 2001, 414, 33-34.

11. Zhai, L.; Berg, M. C.; Fevzi, C.; Kim, Y.; Milwid, J. M.; Rubner, M. F.; Cohen, R. E. Patterned Superhydrophobic Surfaces : Toward a Synthetic Mimic of the Namib Desert Beetle. Nano Lett. 2006, $6,1213-1217$.

12. Zhang, L.; Wu, J.; Hedhili, M. N.; Yang, X.; Wang, P. Inkjet Printing for Direct Micropatterning of a Superhydrophobic Surface : toward Biomimetic Fog Harvesting Surfaces. J. Mater. Chem. A 2015, 3, $2844-2852$.

13. Chen, X.; Ma, R.; Li, J.; Hao, C.; Guo, W.; Luk, B. L.; Li, S. C.; Yao, S.; Wang, Z. Evaporation of Droplets on Superhydrophobic Surfaces : Surface Roughness and Small Droplet Size Effects. Phys. Rev. Lett. 2012, 109, 1-6.

14. Ghosh, A.; Beaini, S.; Zhang, B. J.; Ganguly, R.; Megaridis, C. M. Enhancing Dropwise Condensation through Bioinspired Wettability Patterning. Langmuir 2014, 30, 13103-13115.

15. Mahapatra, P. S.; Gosh, A.; Ganguly, R.; Megaridis, C. M. Key Design and Operating Parameters for Enhancing Dropwise Condensation through Wettability Patterning. Int. J. Heat Mass Transfer 2016, 92, 877-883.

16. Attinger, D.; Frankiewicz, C.; Betz, A. R.; Schutzius, T. M.; Ganguly, R.; Das, A.; Kim, C. J.; Megaridis, C. M. Surface Engineering for Phase Change Heat Transfer : A Review. MRS Energy Sustainability. 2014, 1-40.

17. Ghosh, A.; Ganguly, R.; Schutzius, T. M.; Megaridis, C. M. Wettability Patterning for High-Rate, Pumpless Fluid Transport on Open, Non-Planar Microfluidic Platforms. Lab Chip 2014, 14, 1538-1550.

18. Koukoravas, T. P.; Ghosh, A.; Sinha, P.; Ganguly, R.; Megaridis, C. M. Spatially-Selective Cooling by Liquid Jet Impinging Orthogonally on a Wettability-Patterned Surface. Int. J. Heat Mass Transfer 2016, $95,142-152$. 
19. Koukoravas, T. P.; Mahapatra, P. S.; Ganguly, R.; Megaridis, C. M. Wettability-Confined Liquid-Film Convective Cooling: Parameter Study. Int. J. Heat Mass Transfer 2018, 126, 667-676.

20. Brochard, F. Motions of Droplets on Solid Surfaces Induced by Chemical or Thermal Gradients. Langmuir 1989, 5, 432-438.

21. Zhang, G.; Zhang, X.; Li, M.; Su, Z. A Surface with Superoleophilic-to-Superoleophobic Wettability Gradient. ACS Appl. Mater. Interfaces 2014, 6, 1729-1733.

22. Yin, J.; Li, X.; Yu, J.; Zhang, Z.; Zhou, J.; Guo, W. Generating Electricity by Moving a Droplet of Ionic Liquid along Graphene. Nat. Nanotechnol. 2014, 9, 378-383.

23. Werder, T.; Walther, J. H.; Jaffe, R. L.; Halicioglu, T.; Koumoutsakos, P. On the Water-Carbon Interaction for Use in Molecular Dynamics Simulations of Graphite and Carbon Nanotubes. J. Phys. Chem. B. 2003, 10\%, 1345-1352.

24. Sergi, D.; Scocchi, G.; Ortona, A. Fluid Phase Equilibria Molecular Dynamics Simulations of the Contact Angle between Water Droplets and Graphite Surfaces. Fluid Phase Equilib. 2012, 332, 173-177.

25. Angelikopoulos, P.; Papadimitriou, C.; Koumoutsakos, P. Data Driven, Predictive Molecular Dynamics for Nanoscale Flow Simulations under Uncertainty. J. Phys. Chem. B. 2013, 117, 14808-14816.

26. Chakraborty, M.; Chowdhury, A.; Bhusan, R.; Dasgupta, S. Molecular Dynamics Study of Thermally Augmented Nanodroplet Motion on Chemical Energy Induced Wettability Gradient Surfaces. Langmuir 2015, 31, 11260-11268.

27. Zhang, K.; Wang, F.; Zhao, X. The Self-Propelled Movement of the Water Nanodroplet in Different Surface Wettability Gradients : A Contact Angle View. Comput. Mater. Sci. 2016, 124, 190-194.

28. Liu, Q.; Xu, B. Actuating Water Droplets on Graphene via Surface Wettability Gradients. Langmuir 2015, 31, 9070-9075.

29. Zhang, K.; Wang, F.; Lu, Y. Molecular Dynamics Simulation of Continuous Nanoflow Transport through the Uneven Wettability Channel. AIP Adv. 2018, 8, 015111.

30. Sen, U.; Chatterjee, S.; Ganguly, R.; Dodge, R.; Yu, L.; Megaridis, C. M. Scaling Laws in Directional Spreading of Droplets on Wettability- Confined Diverging Tracks. Langmuir 2018, 34, 1899-1907. 
31. Zheng, Y.; Cheng, J.; Zhou, C.; Xing, H.; Wen, X.; Pi, P.; Xu, S. Droplet Motion on a Shape Gradient Surface . Langmuir 2017, 33, 4172-4177.

32. Zambrano, H. A.; Walther, J. H.; Koumoutsakos, P.; Sbalzarini, I. F. Thermophoretic Motion of Water Nanodroplets Confined inside Carbon Nanotubes. Nano Lett. 2009, 9, 66-71.

33. Oyarzua, E.; Walther, J. H.; Zambrano, H. A. Water Thermophoresis in Carbon Nanotubes: the Interplay between Thermophoretic and Friction Forces. Phys. Chem. Chem. Phys. 2018, 20, 3672-3677.

34. Walther, J. H.; Ritos, K.; Cruz-Chu, E. R.; Megaridis, C. M.; Koumoutsakos, P. Barriers to Superfast Water Transport in Carbon Nanotube Membranes. Nano Lett. 2013, 13, 1910-1914.

35. Rajegowda, R.; Kannam, S. K.; Hartkamp, R.; Sathian, S. P. Thermophoretically Driven Water Droplets on Graphene and Boron Nitride Surfaces. Nanotechnology 2018, 29, 215401.

36. Zhang, L.; Wang, X. Computational Insights of Water Droplet Transport on Graphene Sheet with Chemical Density. J. Appl. Phys. 2014, 115, 194306.

37. Marbach, S.; Dean, D. S.; Bocquet, L. Transport and Dispersion across Wiggling Nanopores. Nat. Phys. 2018, 14, 1108-1113.

38. Secchi, E.; Marbach, S.; Nigues, A.; Stein, D.; Siria, A.; Bocquet, L. Massive Radius-Dependent Flow Slippage in Carbon Nanotubes. Nature 2016, 537, 210-213.

39. N'guessan, H. E.; Leh, A.; Cox, P.; Bahadur, P.; Tadmor, R.; Patra, P.; Vajtai, R.; Ajayan, P. M.; Wasnik, P. Water Tribology on Graphene. Nat. Commun. 2012, 3, 1242.

40. Alejandre, J.; Chapela, G. A. The Surface Tension of TIP4P/2005 Water Model Using the Ewald Sums for the Dispersion Interactions. J. Chem. Phys. 2010, 132, 014701.

41. Cruz-chu, E. R.; Aksimentiev, A.; Schulten, K. Water - Silica Force Field for Simulating Nanodevices. J. Phys. Chem. B. 2006, 110, 21497-21508.

42. Plimpton, S. Fast Parallel Algorithms for Short-Range Molecular Dynamics. J. Comput. Phys. 1995, $117,1-19$.

43. Horn, H. W.; Swope, W. C.; Pitera, J. W.; Madura, J. D.; Dick, T. J.; Hura, G. L.; Head-Gordon, T. Development of an Improved Four-Site Water Model for Biomolecular Simulations: TIP4P-Ew. J. Chem. Phys. 2004, 120, 9665-9678. 
44. Kräutler, V.; Van Gunsteren, W. F.; Hünenberger, P. H. A Fast SHAKE Algorithm to Solve Distance Constraint Equations for Small Molecules in Molecular Dynamics Simulations. J. Comput. Chem. 2001, 22, 501-508.

45. Mayo, S. L.; Olafson, B. D.; Goddard, W. A. DREIDING: A Generic Force Field for Molecular Simulations . J. Chem. Phys. 1990, 94, 8897-8909.

46. Jorgensen, W. L.; Chandrasekhar, J.; Madura, J. D.; Impey, R. W.; Klein, M. L. Comparison of Simple Potential Functions for Simulating Liquid Water. J. Chem. Phys. 1983, 79, 926-935.

47. Deserno, M.; Holm, C. How to Mesh up Ewald Sums. I. A Theoretical and Numerical Comparison of Various Particle Mesh Routines. J. Chem. Phys. 1998, 109, 7678-7693.

48. Ma, M.; Tocci, G.; Michaelides, A.; Aeppli, G. Fast Diffusion of Water Nanodroplets on Graphene. Nature Mat. 2015, 15, 66-72.

49. Cruz-Chu, E. R.; Papadopoulou, E.; Walther, J. H.; Popadic, A.; Li, G.; Praprotnik, M.; Koumoutsakos, P. On Phonons and Water Flow Enhancement in Carbon Nanotubes. Nat. Nanotechnol. 2017, 12, 1106-1108.

50. Humphrey, W.; Dalke, A.; Schulten, K. VMD - Visual Molecular Dynamics. J. Mol. Graphics. 1996, $14,33-38$.

51. Michaud-Agrawal, N.; Denning, E. J.; Woolf, T. B.; Beckstein, O. MDAnalysis: A Toolkit for the Analysis of Molecular Dynamics Simulations. J. Comput. Chem. 2011, 32, 2319-2327.

52. WCCNT. https://github.com/cselab/wccnt, Accessed: 2016-10-29.

53. Frenkel, D.; Smit, B. Understanding Molecular Simulation; Academic Press: San Diego, CA, 2002.

54. Basconi, J. E.; Shirts, M. R. Effects of Temperature Control Algorithms on Transport Properties and Kinetics in Molecular Dynamics Simulations. J. Chem. Theory Comput. 2013, 9, 2887-2899.

55. Ruiz-Franco, J.; Rovigatti, L.; Zaccarelli, E. On the Effect of the Thermostat in Non-Equilibrium Molecular Dynamics Simulations . Eur. Phys. J. E 2018, 41, 80. 


\section{Graphical TOC Entry}

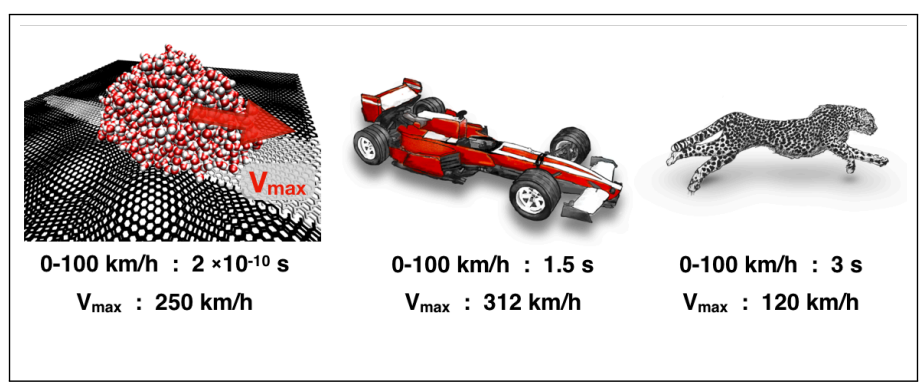

